\title{
Peculiarities of soft gamma-radiation variations in the atmosphere
}

\author{
Aleksei Germanenko ${ }^{1 *}$, Evgenya Mikhalko ${ }^{1}$,Evgeny Maurchev ${ }^{1}$, Boris Gvozdevsky $^{1}$, Igor \\ Yankovsky $^{2}$ \\ ${ }^{1}$ Polar Geophysical Institute, 184209 Apatity, 26A Academgorodok, Russia. \\ ${ }^{2}$ Kabardino-Balkarian State University, 360004, Nalchik, 173 Chernyshevsky St., Russia.
}

\begin{abstract}
Soft gamma-radiation $(20-400 \mathrm{keV})$ arriving from the atmosphere is being monitored by Polar Geophysical Institute (PGI), Apatity, Russia, at several stations located between Spitsbergen and the Causases. In Apatity and Spitsbergen, it has been carried out for ten years already. Detectors of the same type with output integral channels $>20 \mathrm{keV}$ and $>100 \mathrm{keV}$ are used. All the stations record increases in the gammaradiation flux, which are sometimes as high as $100 \%$. These increases occur all the year round, in winter and summer and are recorded at all the stations. A great database on these increases has been used to analyze their characteristics. The detector used in Apatity, is integrated into a complex set carrying out monitoring over the main components of the secondary cosmic rays. According to the earlier experiments, no radioactive contamination has been found in precipitation. The integral and differential data have been analyzed to reveal the peculiarities of the phenomenon studied.
\end{abstract}

\section{Introduction}

The observations made at PGI over the variations of the secondary cosmic rays, have discovered a new phenomenon - the gamma-radiation increase in precipitation. Since the beginning of the observations it has been found that during precipitation the gammaradiation flux increases by tens percent [1]. It is observed all the year round. It has been also revealed that the increases occur only in the electromagnetic component, the charged particles flux variations (electrons, positrons, muons) do not exceed the level of fluctuations of $1-2 \%$, getting lost on this background.

At present, detectors are installed at the stations, such as: Barentsburg (arch. Spitsbergen, $78 \mathrm{~N})$, Apatity (68 N), Tiksi (71 N), Yakutsk (62 N), Houlougaysha peak (50 $\mathrm{N}$ ), Rostov-on-Don (47 N), Neutrino settlement (North Caucasus, $42 \mathrm{~N}$ ). In spite of the differences in geophysical and meteorological conditions, observing everywhere is one and the same effect: precipitation (rain or snow) induces the increase in gamma-radiation by tens percent above the background level recorded before at a good weather. Use is made of scintillation detectors $\mathrm{NaI}(\mathrm{Tl})$ made at PGI, with the energy range of $20-400 \mathrm{keV}$. The detectors are calibrated by the known lines of elements, are placed into lead boxes with

*Corresponding author: germanenko@pgia.ru 
wall thickness of $5 \mathrm{~cm}$, which shield the radiation from soil and surrounding objects. The boxes are opened in top. As other variations of gamma-radiation are observed (for instance, a daily variation of $2-3 \%$ in amplitude), the following threshold has been taken: the increases of the gamma-radiation flux being less than $5 \%$ are not considered as events.

The phenomenon discovered by the authors is being actively studied. In Apatity, they use a complex set to carry out monitoring over all the components of the secondary cosmic rays: nucleon, electron-muon and electro-magnetic components [2,3], as well as monitoring over the gamma-radiation spectrum in the range of $0.2-8 \mathrm{MeV}$. It has been clearly revealed that the energy spectrum of radiation has an exponential form during the increasing, extending to $2.5-3 \mathrm{MeV}$ in the energy range. The energy spectrum of the background radiation in the clear weather has a power form, with a power law index close to -2 . This allows the nature of the soft background radiation in the atmosphere to be identified. It is of a Bremsstrahlung type [4].

\section{A complex set in Apatity and the additional measurements made with the it}

The neutron monitor (NM) station located in Apatity is a base one. Besides a conventional NM, it includes: leadless NM section, a narrow-beam thermal neutrons detector, a charged particles detector $(>5 \mathrm{MeV})$, large and small $\mathrm{NaI}(\mathrm{Tl})$ scintillation gamma-radiation detectors. Their energy range is $0.2-8 \mathrm{MeV}$ and $20-400 \mathrm{keV}$, respectively. This set of instruments is a complex intended for monitoring over all the components of the secondary cosmic rays. The data come to the recorder and are preserved in files.

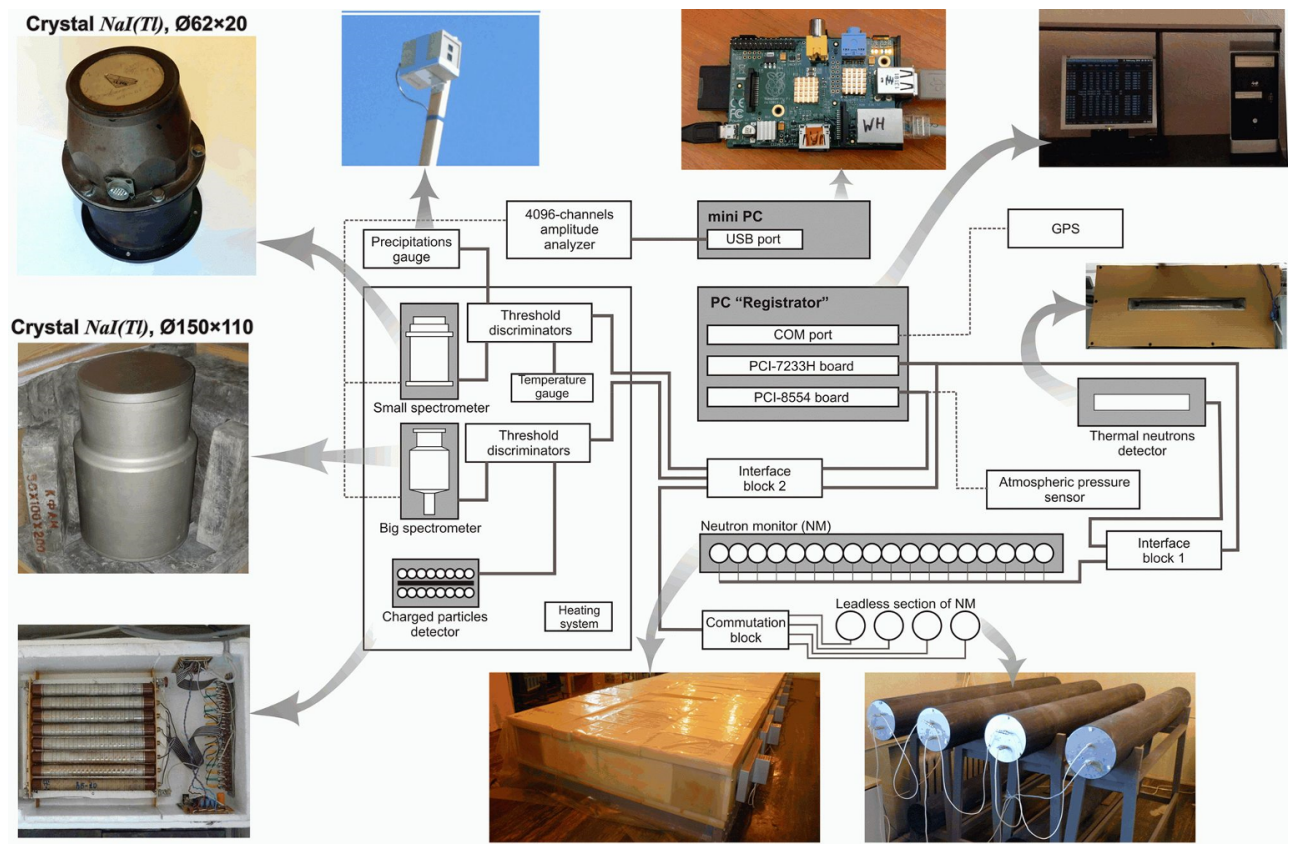

Fig.1. A diagram of a complex set for monitoring over secondary cosmic rays. Besides the detectors defined in the text, the set is equipped with pressure, temperature and precipitation sensors, a GPSsignals receiver for time correction. The blocks "4096-channels analyzer" and "mini-PC" are used to make measurements of the differential spectrum. One spectrum is accumulated for 30 minutes.

The scheme of the complex is given in Fig.1. A large detector makes it possible to carry 
out additional measurements of the differential spectrum of gamma-radiation in the same energy range [2].

It is the measurements of the differential spectrum that allowed exact determination of the upper energy limit of gamma-radiation increases in precipitation, which is $2.5-3 \mathrm{MeV}$. The spectral measurements also show that during increases, the radiation with the exponential spectrum is added to the background flux of gamma-radiation with the power law spectrum. Thus, the energy spectrum of radiation which induces the observed increase in precipitation, greatly differs in form from the background one. It may be supposed that the origin of the additional radiation differs from that of the origin of the background one.

In Apatity, the recording system (Fig.1) includes a precipitation gauge to measure precipitation intensity every minute. Using this instrument, it was clearly determined that the increases start as soon as precipitation starts. The maximal speed of increase is at a moment of maximum precipitation intensity, and the maximum radiation flux comes 30-40 minutes later. It has been also found that there are no radio-nuclides in precipitation [2]. Firstly, it is seen from the differential energy spectrum, in which there are no any lines of elements. Secondly, it follows from the general position of the observing.

In winter, a fresh snow cover is on the roof (over the detector) until, several days later, the wind blows it off the roof. If radio-nuclides were present in a fresh snow cover, the detector would record an increased gamma-radiation flux during those days when the snow cover stayed on the roof over the detector for several days. In fact, however, when snow falling stops, the flux decreases, reaching its background level, with the delay of 30-40 minutes mentioned above. Such fact is not possible in a case of radio-nuclides contamination.
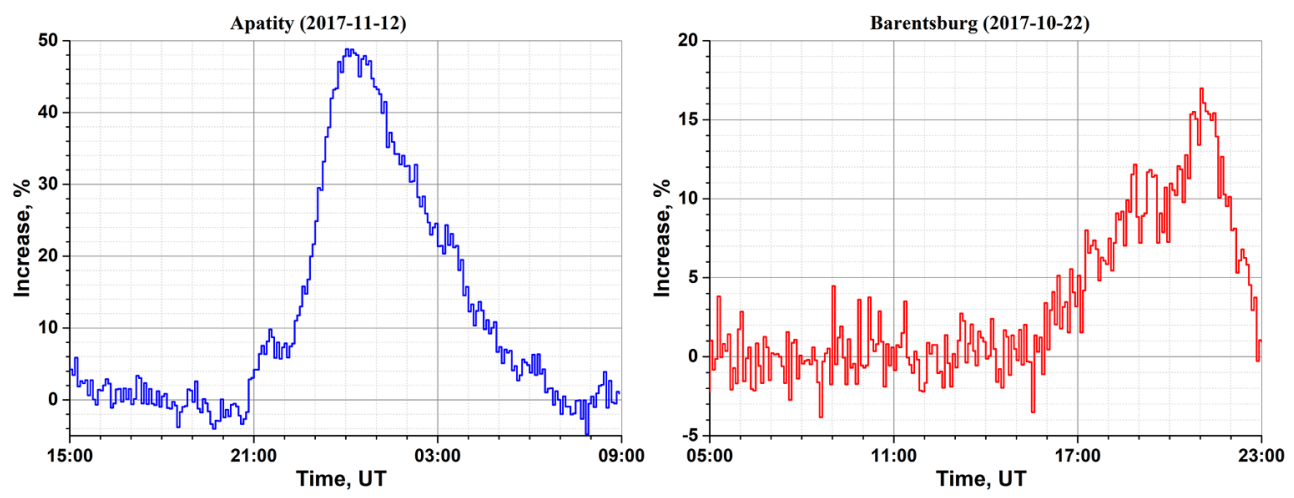

Fig.2. An example of typical increases in gamma-radiation at the stations Apatity (left) and Barentsburg (right). The channel is $>20 \mathrm{keV}$. The flux observed in good weather before is taken as the base level.

\section{Criteria specifying and the obtained results}

Most stations mentioned above came to operate 1-2 years ago, so the statistics obtained is reliable, in terms of accuracy, only at the stations Apatity and Barentsburg. The data quality has been preliminary analyzed to show that the form of the increases profile, as well as their frequency and amplitude depend on the season of the year. So, it was decided to make a more careful and detailed analysis of the increase event characteristics and reveal the events distribution character every month.

More than 1500 events and more than 400 events have been recorded for nine years in Apatity and in Barentsburg respectively. This amount is enough to make a statistical 
analysis of the events. The data recorded over the period mentioned are processed, all the events with the threshold that exceeds that one mentioned above $(5 \%)$, are distinguished, and the following parameters are determined, such as: increase amplitude, increase duration, the front duration (from the background level to 0.8 of the maximum), the greatest speed of increase and speed of decrease after event, and the index proportional to energy released during the period of increase. Then the distribution of these parameters through the months was built for each station. Firstly, the distribution was built of the events through the months during the whole period of observations (Fig.3). Working with the data, the events were qualitatively assessed to show that the amount of the events was less in winter months. The event distribution determined is true for Apatity but is wrong for Barentsburg. In Barentsburg, there is a great failure (the number of events is by an order of magnitude less) in spring (April-June). In Apatity, the number of events is by $20-30 \%$ less in winterspring. The maximum values observed in January and September in Barentsburg, are rather significant, being higher than the statistical fluctuation.

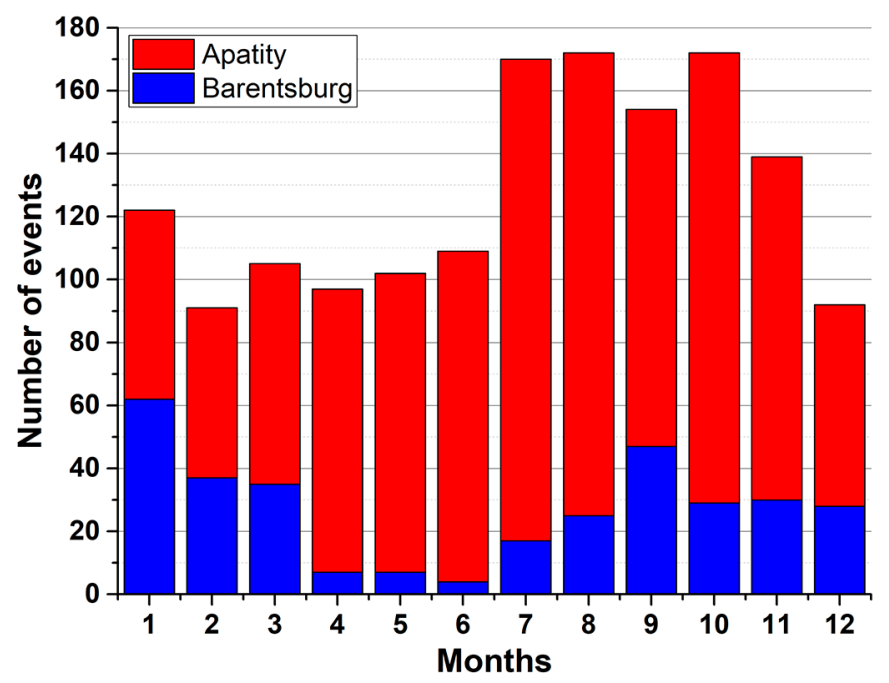

Fig.3. A through-month distribution of the amount of the gamma-radiation increase events recorded at the stations for nine years (2010-2018). The total amount of events recorded in Apatity is 1524, in Barentsburg is 429 .

Figure 4 shows the distribution of events in amplitude: how frequently the increases exceeding the specified amplitude are recorded at the stations. Figure 2 clearly shows that typical increases at the stations differ in amplitude. In Barentsburg, the maximum amplitude is $40 \%$, in Apatity $-100 \%$. So, each station has been provided with the threshold values peculiar for each of the stations. The set of the values was not free. The high threshold would result in small number of events and the distribution would be of low accuracy. So, the first, smaller, threshold (10\% for Barentsburg, and 15\% - for Apatity) is taken as 0.8 of the average one, for all the increases, amplitude; the second threshold is the first doubled. One can say that the first threshold identifies significant events, the second large events. 

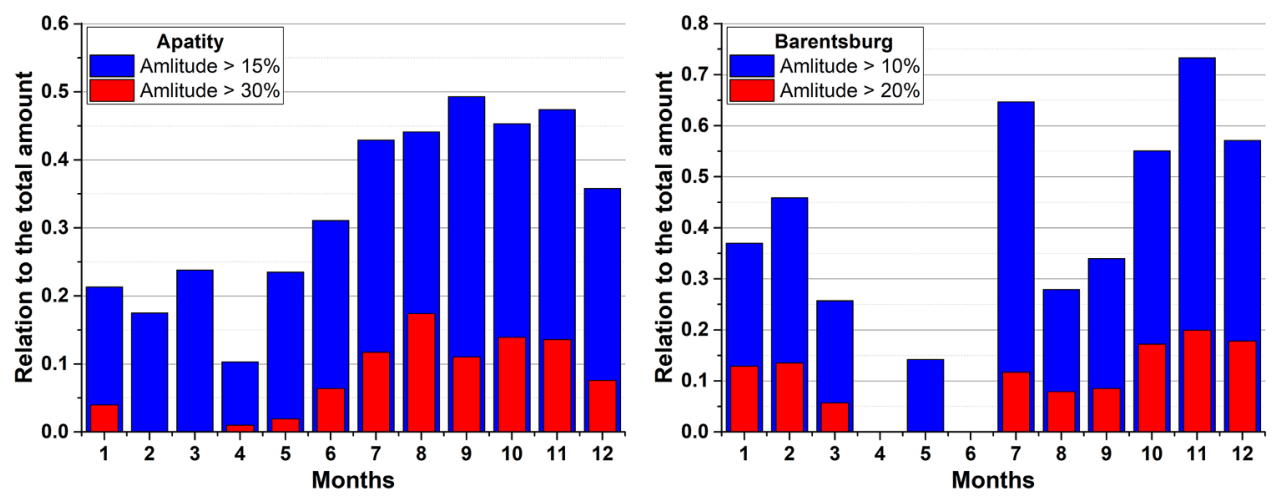

Fig.4. The distribution of the event amplitudes through months. "Relation to the total amount" means a part of the events, which is higher than the specified threshold, of the total amount of events occurred in the month given.

It was pointed out in terms of quality that in a cold season, increases in amplitude are less, and the distribution determined confirms this fact for Apatity rather than for Barentsburg. In fact, in Apatity, there are practically no increases higher than $30 \%$ in cold seasons (including May). The share of significant increases in Apatity in the warm seasons grows more than two times. In Barentsburg, large events do not tend to occur in a warm season, though the maximum share falls at October-December. As for Barentsburg, this period is not considered to be warm: at this period the snow cover is constant already, the temperature is below zero.

Besides the amplitude, increases differ in speed of intensity growth (increase front). There occur events in which the gamma-radiation flux increases so slowly that the event front lasts for many hours, and the beginning and the reaching of the maximum are difficult to determine, and the increase itself looks like a daily variation of a big amplitude. If the threshold is specified to be rather short, it allows us to select increases which are undoubtedly related to precipitation.
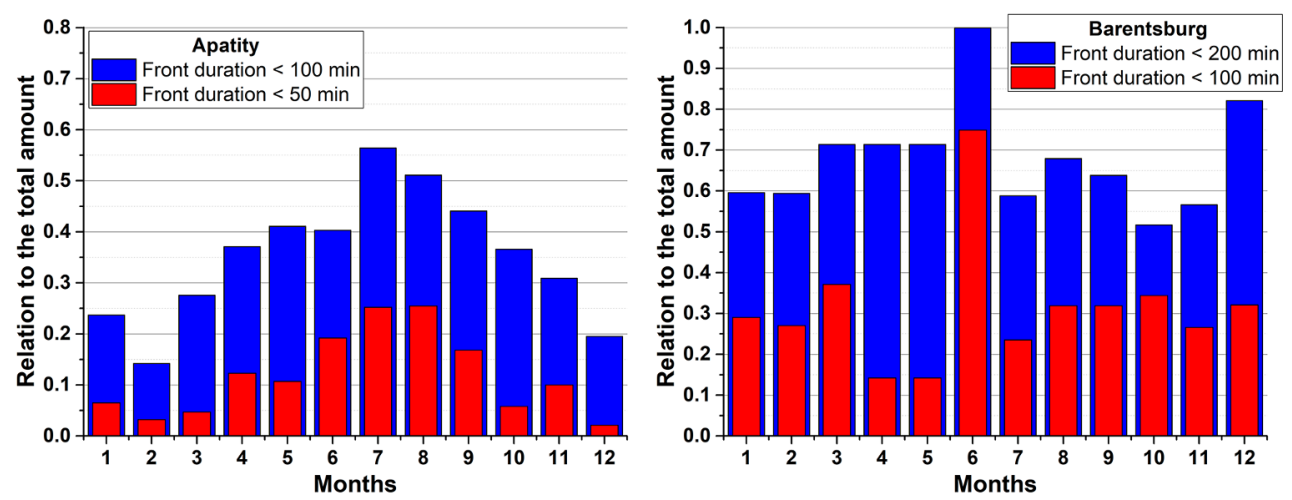

Fig. 5. The distribution of events with a steep leading edge. "Relation to the total amount" means a part of the events, which is higher than the specified threshold, of the total amount of events occurred in the month given.

As we see, a warm season in Apatity is characterized by a noticeable share of increases that begin very sharply; in a cold season, their share decreases at times. As for Barentsburg, there is no clear difference between warm and cold seasons in the steepness of the increase. The increases with an extended or short front have no dominant concentration through 
months.

As the detectors are similar, calibrated and the spectral gamma-radiation characteristics are known, we can calculate the average energy brought by a one quantum through into the detector. It is about $100 \mathrm{keV}$. The threshold $\mathrm{E}_{\text {thld }}$ is specified as $2.5 \times 10^{4}$ quanta. It corresponds to a total amount of the gamma-radiation quanta, which passed into the detector during the event of the average amplitude in Apatity [2]. The threshold $\mathrm{E}_{\text {thld }}$ is specified to be similar for the two stations in order to compare the total energy release of the additional gamma-radiation in the events at the two stations. The distribution of increases with the total energy released higher than the specified threshold $\mathrm{E}_{\text {thld }}$ is given in Fig.6. This distribution reveals an unexpected fact. In spite of the fact that the increase amplitudes in Barentsburg are 1.5-2 times less than those in Apatity, the number of events in which the energy released exceeded the specified threshold, turned out to be one and a half time greater than that in Apatity! The reason of such a seeming contradiction is in that increases in Barentsburg is sufficiently longer, which compensates a small value of amplitude.
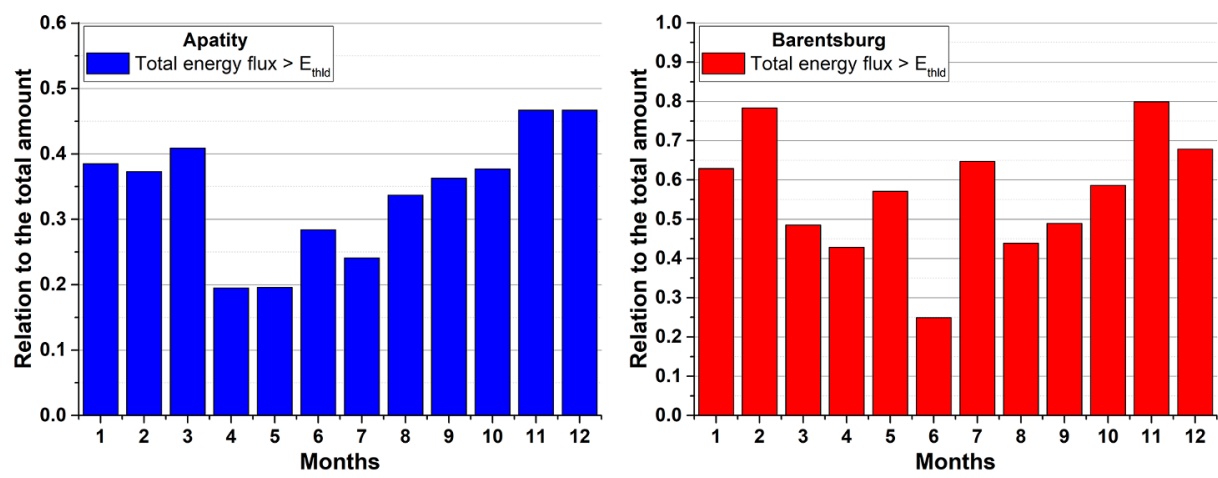

Fig.6. The distribution of the events according to the amount of the additional energy released during the event. "Relation to the total amount" means a part of the events, which is higher than the specified threshold, of the total amount of events occurred in the month given.

\section{Discussion}

Using an extensive database accumulated at the stations Apatity and Barentsburg, the events were analyzed and their distribution through months by the different characteristics was found. The qualitative conclusions made earlier on the dependence of the event characteristics on the seasons turned out to be rather approximated. The distribution of the total amount of events through months in Apatity turned out to be not so uneven; the differences were as small as $20-30 \%$, while in Barentsburg, a substantial failure was found in April - June period.

The distributions of amplitude have confirmed the qualitative assessment concerning the fact that large increases occur in summer, but it would be more correctly to say that these are shifted to the second half of the year, to July-December. It is true for the two stations, though there are some differences. The distribution of increases in the front duration shows that in Apatity, increases tend to concentrate, with the sharp beginning in the warm season. In Barentsburg, such trend is not observed. The distributions of increases in total energy which released additionally during increase, tuned out to be close in form at the two stations. In Barentsburg, the part of the events with the energy release greater than that specified, turned out to be greater than that in Apatity.

The set of the parameter "total energy released during increase" is the most important in 
distribution. The calculation of this value and calculating of the distribution have shown that the events at these stations are approximately equal in the additional energy released (the difference is two times less). Hence, the energy of the process inducing the increase at the stations, changes slightly from both season to season and the place of observation, in spite of the differences in form of the increase profiles and their amplitude. It seems that gamma-radiation increases are due to the global factor effective everywhere in the atmosphere. This distribution also suggests that the hypothesis of the radon nature of the increases is wrong. The station Barentsburg is located on the bank of the Greenfjord, Spitsbergen is the zone of permafrost. These factors could have decreased the radon emission into the atmosphere, and, as a result, have decreased the energy released. But quite other effect is observed: in Barentsburg, on the average, the events release much more energy than in Apatity.

\section{Conclusions}

Based on a huge database on the increase events in Apatity and Barentsburg, an analysis has been made of the distribution of the events through months, taking different characteristics into account. In spite of the fact that the stations are located in the polar region, there are found significant differences in the event distribution.

At the same time, the event distributions in a very important factor, such as the total gamma-radiation energy released during the increase, are rather close in form and values. This argument is very significant in favor of the hypothesis about a single global mechanism of the gamma-radiation generation during precipitation.

\section{References}

1. A.V. Germanenko, Yu.V. Balabin, E.V. Vashenyuk, B.B. Gvozdevsky, L.I. Schur, Astrophys. Space Sci. Trans., 7, 471 (2011)

2. J.V. Balabin, A.V. Germanenko, B.B. Gvozdevsky, E.V. Vashenjuk, Geomagnetism and aeronomy, 54, 376 (2014)

3. Balabin Yu.V., Germanenko A.V., Gvozdevsky B.B., Vashenyuk E.V., Bulletin of the Russian Academy of Sciences: Physics, 77, 572 (2013)

4. S. Hayakawa, Cosmic Ray Physics. Nuclear and Astrophysical aspects (WileyInterscience, New York, 1969) 